\title{
Estimation of interfacial fracture toughness based on progressive edge delamination of a thin transparent coating on a polymer substrate
}

\author{
S. Tarasovs ${ }^{\mathrm{a}}$, J. Andersons ${ }^{\mathrm{a}, *}$, Y. Leterrier ${ }^{\mathrm{b}}$ \\ ${ }^{a}$ Institute of Polymer Mechanics, University of Latvia, Rìga LV 1006, Latvia \\ ${ }^{\mathrm{b}}$ Laboratoire de Technologie des Composites et Polymères (LTC), Ecole Polytechnique Fédérale de Lausanne (EPFL), \\ CH 1015 Lausanne, Switzerland
}

Received 9 October 2009; received in revised form 18 January 2010; accepted 19 January 2010

Available online 13 February 2010

\begin{abstract}
Evaluation of interfacial toughness of sub-micron-thickness layers deposited on a ductile substrate is a challenging task which has motivated different experimental approaches. Fragmentation testing was used in the present study as a means of interface characterization of a silicon-nitride-coated polyimide substrate. During the test, after an initial rapid segmentation-cracking phase, the coating fragments developed edge delaminations which propagated in a stable manner with further increase in the applied strain. The debonding process was modelled by the finite element method incorporating a cohesive zone at the front of the interfacial crack. The edge cracks were found to be dominated by mode II loading. By fitting the predicted delamination evolution to the experimental data for coating fragments of differing geometry, the mode II critical energy release rate was estimated at $30 \mathrm{~J} \mathrm{~m}^{-2}$.
\end{abstract}

(C) 2010 Acta Materialia Inc. Published by Elsevier Ltd. All rights reserved.

Keywords: Coatings; Adhesion; Tension test; Toughness; Finite element analysis

\section{Introduction}

Polymer foils coated with structured inorganic thin layer stacks are becoming ubiquitous in a variety of applications, e.g., flexible electronics. An appropriate level of adhesion between the polymer substrate and the inorganic layers has to be ensured so that the mechanical integrity and functionality of the stack are not compromised during service. Sub-micron thickness of the layers hampers experimental evaluation of adhesion in terms of fracture mechanics parameters, because introducing an interfacial crack and following its development with load becomes complicated; see e.g., Refs. [1,2]. The present study uses an instrumented fragmentation test to estimate the interfacial fracture toughness of a thin coating on a polymer film.

\footnotetext{
* Corresponding author. Tel.: +371 6754427; fax: +371 67820467.

E-mail address: janis.andersons@pmi.lv (J. Andersons).
}

In the fragmentation test, the development of damage patterns in brittle coatings is analyzed as a function of the applied substrate tensile strain [3]. After an initial stage of intensive accumulation of cohesive cracks, the coating becomes segmented into fragments of width roughly comparable with the stress transfer length, and the fragmentation rate decreases markedly. Lateral compressive stresses that develop in the fragments due to Poisson contraction of the substrate can be released by local buckling-driven delamination [4-6]. Stress concentration at the fragment edges, formed by the cohesive cracks, may also cause debonding [7-9]. Progressive detachment of the coating finally leads to saturation of the fragmentation process.

Several features of the coating/substrate interface can be derived from the fragmentation test results. The interfacial shear strength (IFSS) can be evaluated from a statistical analysis of the coating strength and fragment geometry at saturation, see e.g., Ref. [4]. Since testing up to saturation is not always practical, an alternative to characterize the overall mechanical efficiency of the interface was proposed 
in Ref. [10]. In this approach, an effective stress transfer length, evaluated using the experimentally determined dependence of cohesive crack density on the applied strain in an advanced fragmentation stage, was shown to correlate with the IFSS. Making use of the stress-strain diagrams of plain and coated polymer foil, stretched up to coating detachment, the interface fracture energy was estimated $[7,8,11]$. Energy balance analysis of localized buckling-driven delaminations appearing in coating fragments during the test also provided an estimate of the critical interfacial energy release rate (ERR) [5]. Note, however, that the fracture mechanics characteristics mentioned above do not separate the interfacial ERR into the basic modes. By contrast, mode II fracture toughness of the interface was derived in Ref. [9], based on edge stress analysis and the assumption that the initiation of edge delamination coincides with the appearance of buckles. A cohesive zone model of interface was applied in Ref. [12] to evaluate simultaneously both the adhesive fracture toughness of the interface and the cohesive fracture toughness of the coating, using experimentally determined dependence of coating crack density on the applied strain.

The mechanics of delamination of thin film strips has been established for elastic constituents $[13,14]$ and in the presence of yielding in one of the constituents [15]. In fragmentation tests, coating fragments are usually delaminating at relatively high strains, when the polymer substrate is yielding. A process zone model, implemented in numerical analysis via cohesive elements, provides a means of evaluating the ERR spent on decohesion [15-17]. Such a model allows accurate simulation of the true loading path during the delamination process, which is a critical issue for problems with significant plastic deformation. The accuracy of the numerical approach, hence of the computed ERR values is ensured by comparison of the simulation with the experimental data.

Several experimental techniques are available to quantify the extent of coating delamination. Atomic force microscopy provides a means of accurate mapping of the changes in relative elevation of the coating surface that are related to the evolution of debonds $[6,18,19]$. Ultrasonic force microscopy, being sensitive to local variation in mechanical compliance, has been used to identify locations of impaired adhesion [19]. However, these methods may be difficult to apply for in situ observations during active tension, necessitating interruptions in the mechanical test. Optical methods, based on interferometry (see e.g., Refs. [20,21]), appear particularly suitable for fragmentation tests, owing to relative ease of in situ application.

The present study concerns the evaluation of interfacial adhesion in a silicon-nitride-coated polyimide substrate $\left(\mathrm{SiN}_{x} / \mathrm{PI}\right)$ system by means of fragmentation tests, from the analysis of coating edge delamination. White light interference patterns were used to assess the evolution of delamination with applied strain, and finite element analysis incorporating a cohesive zone model was used to establish the critical interfacial ERR and strength.

\section{Experimental}

A $125-\mu \mathrm{m}$-thick polyimide (PI) substrate $\left(\mathrm{Upilex}^{\circledR} \mathrm{S}\right.$, UBE) was used. The PI foils were annealed at $120^{\circ} \mathrm{C}$ for 30 min. before deposition of $\mathrm{SiN}_{x}$ coatings by plasma enhanced chemical vapor deposition at a temperature of $200{ }^{\circ} \mathrm{C}$. Five different coating thicknesses $(100,200,400$, 600 and $800 \mathrm{~nm}$ ) were investigated. The use of silicon nitride films has become the industrial standard in solar cell production $[22,23]$. It has been demonstrated that the optical properties of $\mathrm{SiN}_{x}$ films can be easily controlled by adjusting the deposition parameters, and a wide range of refraction index values can be attained (from 1.9 to 3.0 or higher for $600 \mathrm{~nm}$ wavelength) $[22,24]$. Optical properties of the coating and the substrate used in the current study, shown in Fig. 1, were determined by spectro-ellipsometry.

Young's modulus $E$ of the $\mathrm{SiN}_{x}$ layers was determined by means of nano-indentation tests, as detailed elsewhere [25], and was found to be equal to $100 \pm 10 \mathrm{GPa}$. Their Poisson coefficient was assumed to be equal to 0.26 [26]. The tensile properties of the PI substrate were determined from tensile tests using dog-bone-type samples of a reduced section equal to $6 \times 50 \mathrm{~mm}^{2}$. Tests were carried out at a strain rate equal to $1.7 \times 10^{-3} \mathrm{~s}^{-1}$ at ambient temperature by means of a universal tensile testing machine (UTS Testsysteme load frame) equipped with a $1000 \mathrm{~N}$ load cell. Fig. 2 shows the tensile behavior of the polymer substrate. Its Young's modulus and Poisson coefficient were found to be equal to $5.35 \mathrm{GPa}$ and 0.25 , respectively. The residual in-plane coating stress was evaluated from the radius of curvature of the coated film measured at $22{ }^{\circ} \mathrm{C}$ and $50 \%$ relative humidity. It was found to be compressive and decreasing in absolute value from $-340 \mathrm{MPa}$ for the $100-\mathrm{nm}$-thick coating to $-200 \mathrm{MPa}$ for the 600- and 800-nm-thick coatings [27].

Fragmentation tests under uniaxial loading were performed at ambient temperature on $50 \times 5 \mathrm{~mm}^{2}$ rectangular samples using a miniature tensile tester with displacement control (Minimat, Rheometric Systems) in situ in an optical microscope (Olympus BX60). The microscope was equipped with a coupled-charge device camera (Soft Imaging Systems ColorView II) for non-contact video extensometry of specimen strain with accuracy better than $10^{-4}$ in strain values. This method eliminated frame compliance and clamp slippage problems. It was verified that the possible influence of ink markers used for extensometry on localized specimen strain was negligible, since their dimensions, typically $100 \mu \mathrm{m}$, were very small compared with the sample width, equal to $5 \mathrm{~mm}$. Coating damage was analyzed at selected strain levels under reflected white halogen light.

Fig. 3 shows the fragmented morphology of the investigated $\mathrm{SiN}_{x}$ coatings under $\sim 10 \%$ tensile strain. A dense array of cohesive cracks is evident for all thicknesses, with systematic occurrence of branching with the exception of the thinnest, $100 \mathrm{~nm}$, coating. Transverse buckling is also visible, with a marked influence of coating thickness on buckle size and density. Numerous buckles with length of the order of 

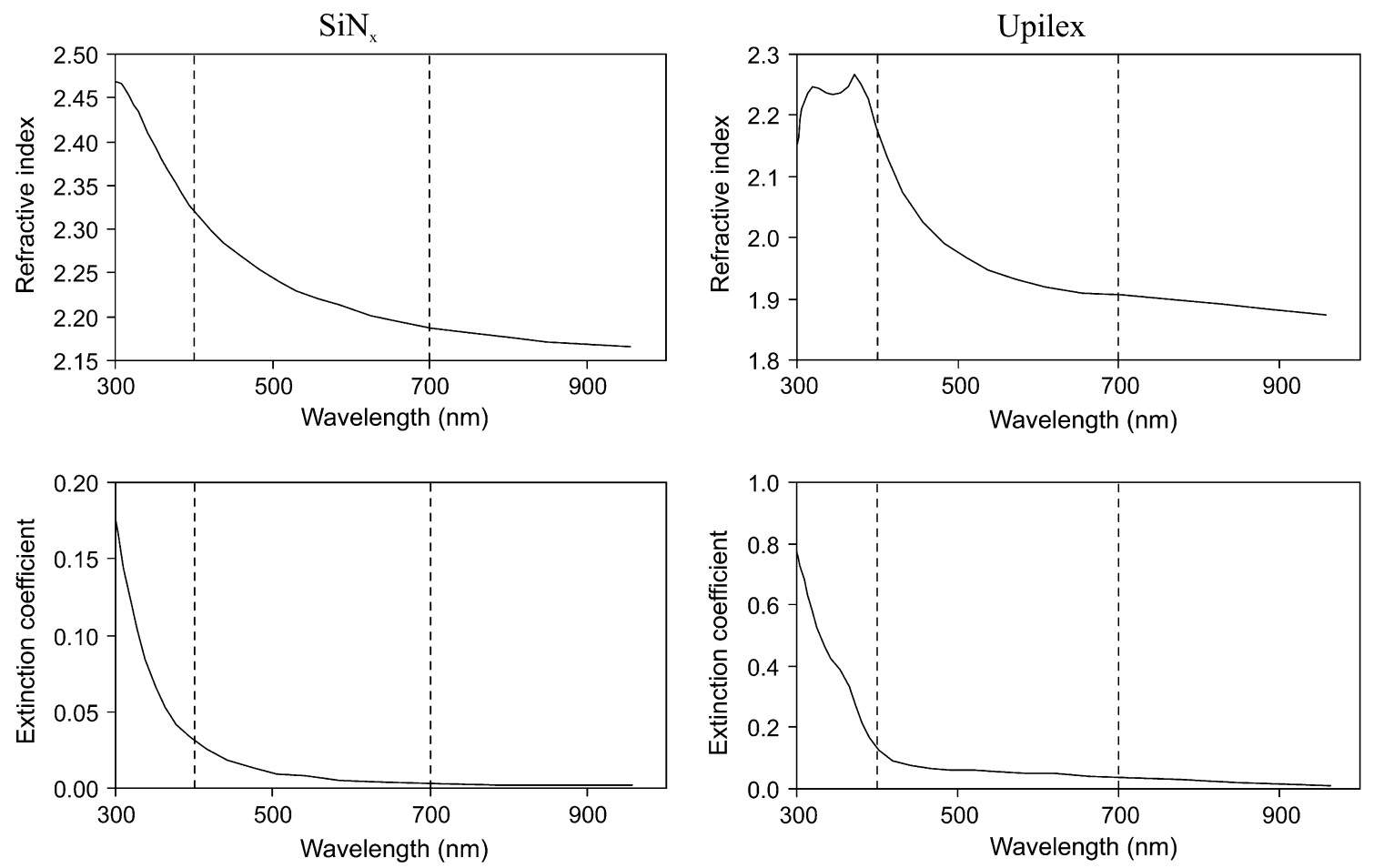

Fig. 1. Refractive indices and extinction coefficients of the 200-nm-thick $\mathrm{SiN}_{x}$ coating and the PI substrate. Dashed lines show the visible light range.

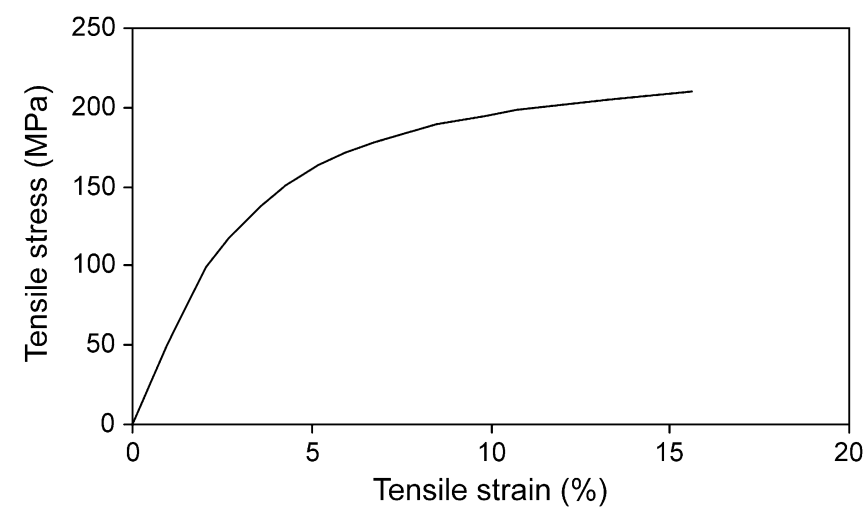

Fig. 2. Stress-strain diagram of the PI substrate in uniaxial tension.

$1 \mu \mathrm{m}$ are present in the 100 -nm-thick coating. Many fewer buckles are present in the thicker coatings, and in this case their length is of the order of $100 \mu \mathrm{m}$. As shown in Fig. 4, the larger buckles give rise to interference patterns with bright and dark Fizeau fringes. One also notices the occurrence of a bright fringe along the fragment edges due to edge delamination. The following model focuses on edge delaminations. In this case, the delaminated profile is flat enough for the interference theory of layered media with parallel interfaces to be applicable (Appendix A).

\section{Model of edge delamination of a coating fragment}

\subsection{Elementary analysis}

Consider a coating fragment of width $w$ adhering to a substrate subjected to a tensile strain $\varepsilon$ parallel to the width of the fragment. Delamination is observed at a relatively advanced fragmentation stage when the length of the tensile stress recovery zone $\lambda$ exceeds the fragment width, $\lambda>w$. As a result, the applied strain is not fully transferred to the coating fragment, and the tensile stress distribution can be approximated as [10]

$\sigma=\frac{\sigma_{0}}{2 \lambda^{2}} x(w-x)$

with the origin of the $x$ axis taken at the fragment edge, and $\sigma_{0}=E \varepsilon$ denoting the far-field coating stress. According to elastic shear lag models, $\lambda$ is interpreted as the distance from a coating crack in the loading direction at which the coating stress reaches the value of $\sigma=(1-1 / e) \sigma_{0} \approx 0.63 \sigma_{0}$ for vanishing crack density.

The strain energy accumulated in the coating fragment per unit length under tensile stress $\sigma$ (Eq. (1)) reads, to a first approximation, as

$U=\int_{0}^{w} \frac{\sigma^{2} h}{2 E} d x=\frac{\sigma_{0}^{2} h w^{5}}{240 E \lambda^{4}}$

With the development of edge delamination, the width of the bonded part of the fragment is reduced as follows:

$w=w_{0}-2 a$

where $a$ is the edge delamination length, and $w$ and $w_{0}$ stand for the current and initial width of the bonded part of fragment, respectively. The strain energy accumulated in the coating and substrate in the vicinity of the debond is partially released by propagating delamination. For simplicity and tractability, it is assumed that only the strain 

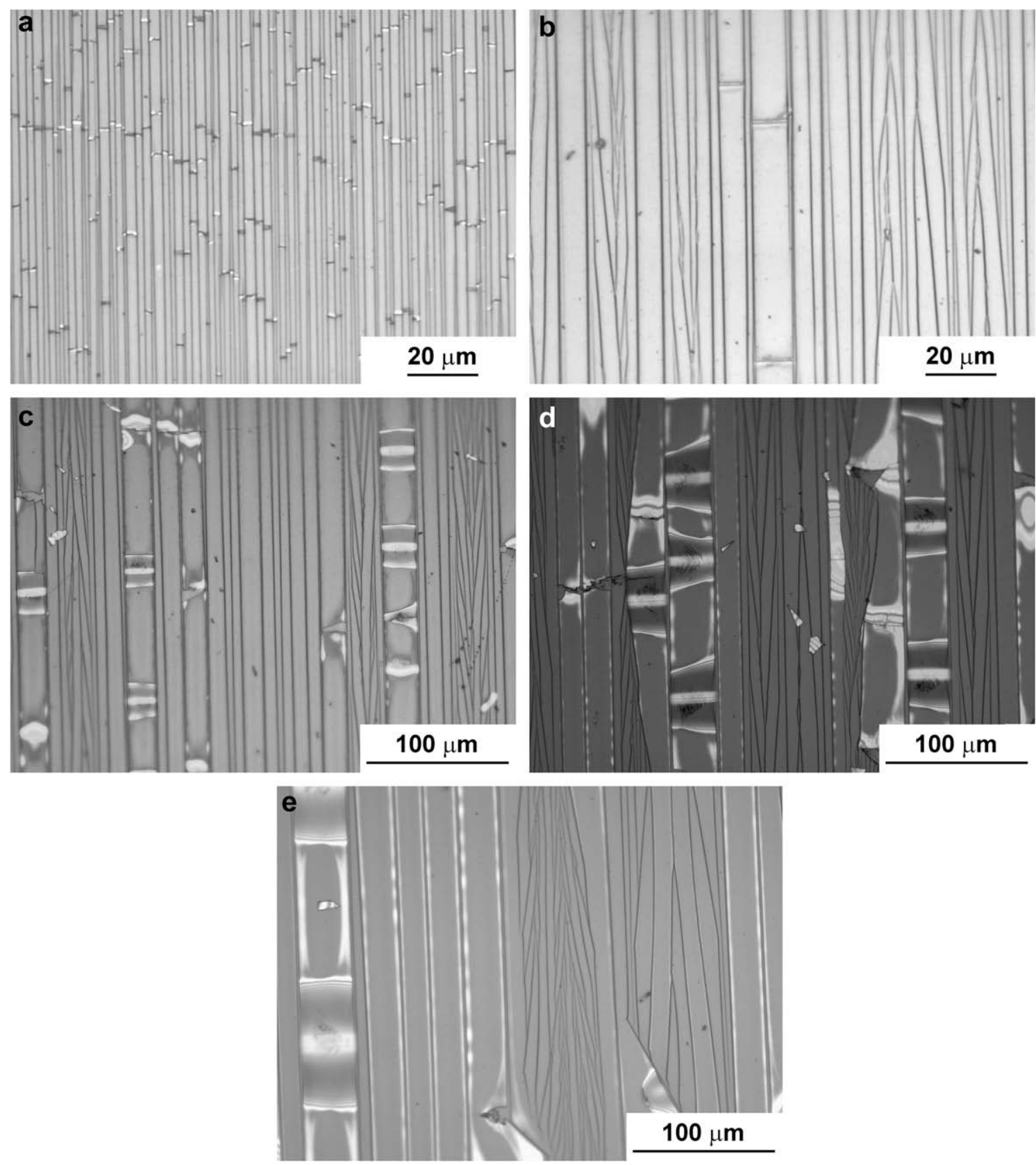

Fig. 3. Fragmented morphology of $\mathrm{SiN}_{x}$ coatings on a PI substrate: (a) $100-\mathrm{nm}$ coating at $12 \%$ strain; (b) 200 -nm coating at $12 \%$ strain; (c) $400-\mathrm{nm}$ coating at 10\% strain; (d) $600-\mathrm{nm}$ coating at 10\% strain; (e) $800-\mathrm{nm}$ coating at $10 \%$ strain. Notice that the magnification is different for the different thicknesses.

energy of the coating drives debonding. Then the ERR $G$ associated with edge delamination is obtained from Eqs. (2) and (3) as follows:

$G=-\frac{\partial U}{\partial a}=\frac{\varepsilon^{2} E h}{24 \lambda^{4}}\left(w_{0}-2 a\right)^{4}$

The delamination onset strain is obtained from Eq. (4) as the strain at which ERR reaches its critical value $G_{c}$ at $a=0$ :

$\varepsilon_{\text {onset }}=2\left(\frac{\lambda}{w_{0}}\right)^{2} \sqrt{\frac{6 G_{c}}{E h}}$

The debond propagation is stable because, according to Eq. (4), the ERR is a decreasing function of crack length at a fixed applied strain. For $\varepsilon>\varepsilon_{\text {onset }}$, the length of edge delamination is given by

$a=\frac{1}{2}\left(w_{0}-\left(\frac{24 G_{c}}{E h}\right)^{1 / 4} \frac{\lambda}{\sqrt{\varepsilon}}\right)$

\subsection{Numerical modelling}

In order to reflect accurately the elastic-plastic response of the matrix and the progressive debonding of the coating, a numerical analysis was carried out using the commercial finite element method (FEM) ABAQUS code. A two- 


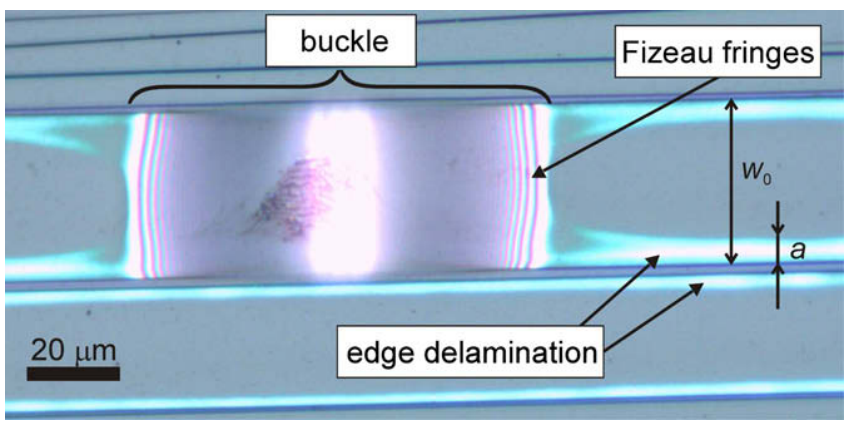

Fig. 4. Fizeau fringes observed at debonded parts of coating fragments due to buckling and edge delaminations of a $800 \mathrm{~nm}$ thick coating at $8 \%$ tensile strain.

dimensional plain strain finite element model was used to simulate coating detachment, with the substrate being modelled as elastic-plastic and the coating as linear elastic material. Linear triangular elements were used for the substrate and quadratic rectangular for the coating. A cohesive zone model for delamination was applied, where the coating was bonded to the substrate by a layer of cohesive elements with a bilinear traction-separation law. Compressive residual stresses of magnitudes reported in Section 2 were induced in the coating by a thermal loading step preceding the mechanical loading step. In fact, calculations showed that their influence on the solution was relatively limited for the considered material system. To improve the convergence rate of the solution, the stabilization procedure available in ABAQUS with dissipated energy fraction equal to $5 \times$ $10^{-5}$ was applied.

\section{Interference patterns due to delamination of a thin coating}

The interference pattern in the reflected light (Fizeau fringes, Figs. 3 and 4) was caused by the presence of an air gap beneath the delaminated part of the coating. The actual pattern was related to the length and opening profile of the delamination: a number of fringes formed at the front of the steeply opening buckling-driven delamination (Fig. 4), whereas only one bright fringe was observed for edge delaminations that typically exhibited both smaller and smoother elevation of the debonded coating $[18,19,28]$.

For small angles between the delaminated coating and the substrate expected for edge delamination, the interference pattern can be assessed by means of the theory of layered media with all the interfaces being parallel, and light incidence normal to the surface (see Appendix A). In order to relate the length of the edge delamination to the extent of the bright fringe at the fragment edge, the intensity of the reflected light was computed as a function of the thickness of the air gap. Polynomial fits of the optical data shown in Fig. 1 within the visible light range were used for calculations (fifth-order polynomials were employed). The reflectance of the bonded film was calculated using expression (A.3) whereas for delaminated coating Eq. (A.4) was used, where the air gap constituted the second layer (hence $n_{3}=n_{1}$ ). The intensity of the light reflected from bonded and from delaminated coating was computed using Eq. (A.7) and the spectrum of a halogen lamp (see e.g., Ref. [29]). The intensity of the reflected light $I$ as a function of the air gap thickness, normalized by the intensity of the light reflected from the bonded film $I_{b}$, is shown in Fig. 5 for the 800-nm coating (for other thicknesses the results are qualitatively similar).

As expected, for a very small air gap the reflectance of the delaminated coating is equal to the reflectance of the bonded film. As a consequence, the exact position of the delamination front cannot be determined from the reflectance contrast. Nevertheless, the interference calculations show that the intensity of the reflected light increases quite steeply with the air gap thickness; e.g., for a $20 \mathrm{~nm}$ gap the brightness exceeds that of a bonded film by $10 \%$.

\section{Results and discussion}

The analysis of edge delamination commences with the assumption that the extent of the interference fringe along the fragment edge virtually coincides with the respective debond length. This assumption is validated with the relatively steep variation in reflectance with interfacial crack opening, established above. Having determined the relevant mechanical characteristics of the interface, the consistency of the assumption with the numerical and experimental results is verified by employing the FEM-predicted geometrical characteristics of delamination profiles in analysis of the interference patterns.

Well-developed delaminations (as revealed by the interference fringes at the fragment edges) were observed for specimens with the thicker coatings of 400,600 and $800 \mathrm{~nm}$. Delaminations initiated at a tensile strain of $\sim 4 \%$ for the $800-\mathrm{nm}$ coating and at higher strains for the coatings with smaller thicknesses. At high strain, the delamination length at the edges of wide fragments was in the range 2-6 $\mu \mathrm{m}$. Detailed observations of the appearance and propagation of edge delamination suggested that

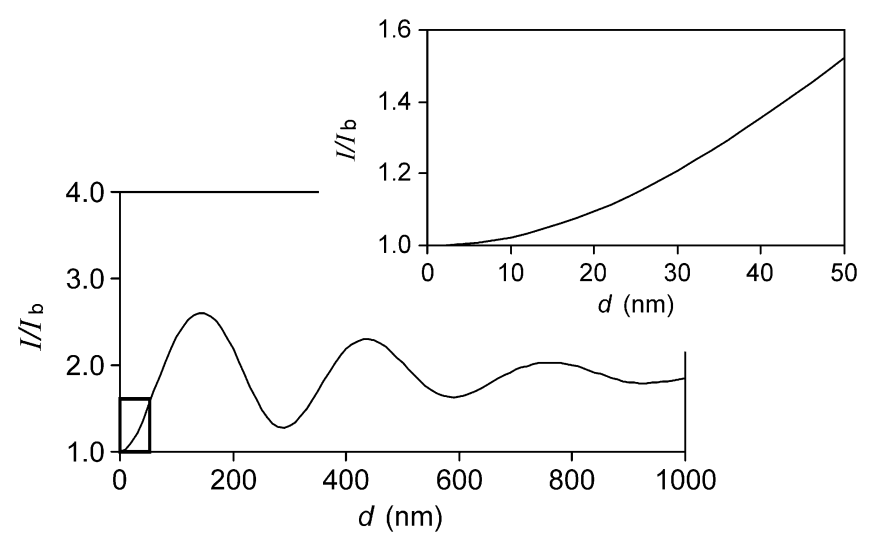

Fig. 5. The intensity of the light reflected from delaminated coating $800 \mathrm{~nm}$ thick as a function of the air gap thickness (the intensity values are normalized by the intensity of the light reflected from bonded film, $I_{b}$ ). 
debonding started at lower strains for thicker coatings, and, for a coating of given thickness, for wider fragments. This was in qualitative agreement with the effect of fragment width and thickness on $\varepsilon_{\text {onset }}$ (Eq. (5)) and on delamination length $a$ (Eq. (6)). For quantitative correlations, the stress transfer length $\lambda$ and the interfacial critical ERR $G_{c}$ have to be evaluated. The former was estimated as $\lambda \sim 30 h$, as was obtained in Ref. [10] for a similar $\operatorname{SiN}_{x} /$ PI foil (differing in that the substrate was Kapton VN) with $h \geqslant 200 \mathrm{~nm}$.

The delamination lengths of selected fragments were determined as illustrated in Fig. 4, from the extent of the bright band at a fragment edge. Relatively wide fragments which, once formed, survived further loading without developing cohesive cracks, were identified in the micrographs. The delamination length was measured at several locations along the fragment for two different fragment widths and two coating thicknesses $(600$ and $800 \mathrm{~nm})$ at three load levels corresponding to $8 \%, 9 \%$ and $10 \%$ applied tensile strain. The delamination length of the 400-nm-thick coating was too small to warrant sufficient accuracy, and this coating thickness was disregarded in the following analysis.

The delamination length as a function of applied strain is shown in Fig. 6 for the studied fragments that comply with the applicability condition of the elementary analysis, $\lambda>w_{0}$. A first estimate of $G_{c}$ at $11 \mathrm{~J} \mathrm{~m}^{-2}$ was obtained by fitting Eq. (6) to the debond data of the thicker, $800 \mathrm{~nm}$, fragment. Eq. (6) was further used to predict the development of edge delamination in the thinner, $600 \mathrm{~nm}$, fragment. The respective theoretical relations plotted in Fig. 6 with solid lines appear to agree reasonably well with the test data, suggesting that the elementary analysis of delamination captures correctly the essential features of the process. However, the accuracy of the above estimate of $G_{c}$ is questionable owing to the simplifying assumptions used in the analysis. The application of a cohesive zone model should provide a more accurate and detailed treatment of energy dissipation due to decohesion.

A cohesive zone model of delamination implies an appropriate selection of the traction-separation law. It

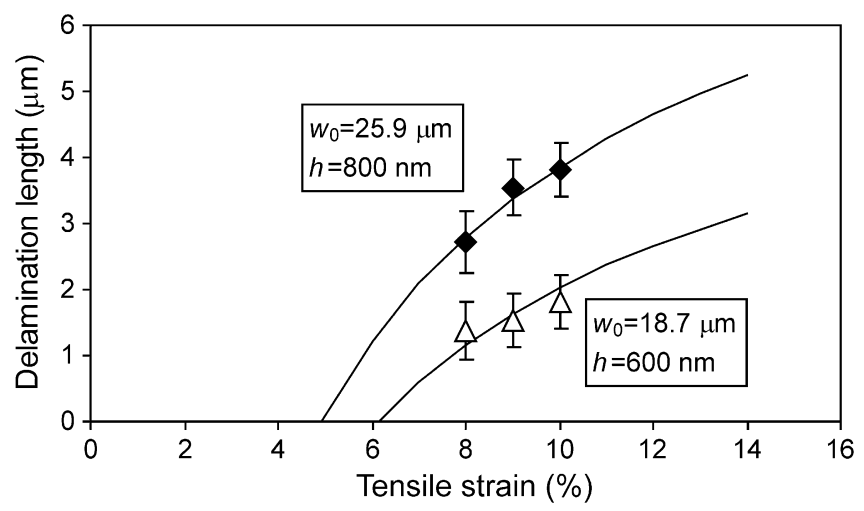

Fig. 6. Experimental (dots) and estimated by Eq. (6) (lines) edge delamination length of coating fragments 600 and $800 \mathrm{~nm}$ thick with different widths $w_{0}$. has been demonstrated that, for reasonably compliant materials, the actual shape of the softening law has a minor effect [30]. The simplest bilinear relation was thus chosen in the present work, with the strength of the element (maximum normal or tangential traction on element faces) $\tau$ and the interfacial ERR $G_{a}$ as the two main parameters. Since the area under the traction-separation curve is equal to $G_{a}$, the element strength and maximum opening (sliding) displacement of the element $\delta$, reached when tractions on element faces are reduced to zero, are related as

$\tau=2 G_{a} / \delta$

The FE analysis revealed that the debonding process was not sensitive to the characteristics of mode I cohesive elements, as mode II loading played the leading role in the problem considered. This was further confirmed by the mode mixity, defined for the cohesive elements as $\tan ^{-1}\left(\sigma_{\tau} / \sigma_{n}\right)$ [1] (where $\sigma_{\tau}$ and $\sigma_{n}$ stand for the tangential and normal tractions at the interface) varying from $80^{\circ}$ at the beginning of the fracture process zone to $70^{\circ}$ at the end of it. Several combinations of critical ERR and interface strength were applied in simulations to determine the combination that provided the best agreement of predicted delamination length with the experimental data. The delamination length in the FE model was determined as the distance from the fragment edge to the end of the fracture process zone where cohesive elements were completely separated, as shown in Fig. 7. The modelling results shown in Fig. 8 were obtained for an interface shear strength equal to $250 \mathrm{MPa}$ and an adhesive mode II ERR of $30 \mathrm{~J} \mathrm{~m}^{-2}$. According to Eq. (7), the critical sliding separation of the cohesive elements was equal to $240 \mathrm{~nm}$. A relatively close agreement with the experimental data for differing fragment widths and thicknesses is seen.

FEM computations with the same interface properties for thinner coatings predicted an increase in delamination onset strain and a decrease in the extent of delamination

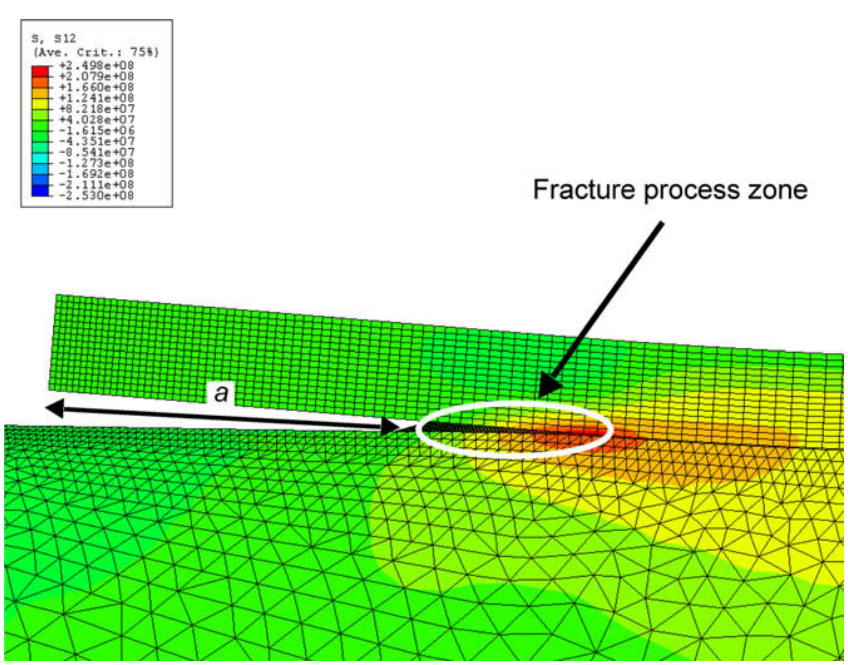

Fig. 7. A fragment of the finite element mesh for 600-nm coating. Delamination of length $a$ and the fracture process zone are shown at $8 \%$ tensile strain. 

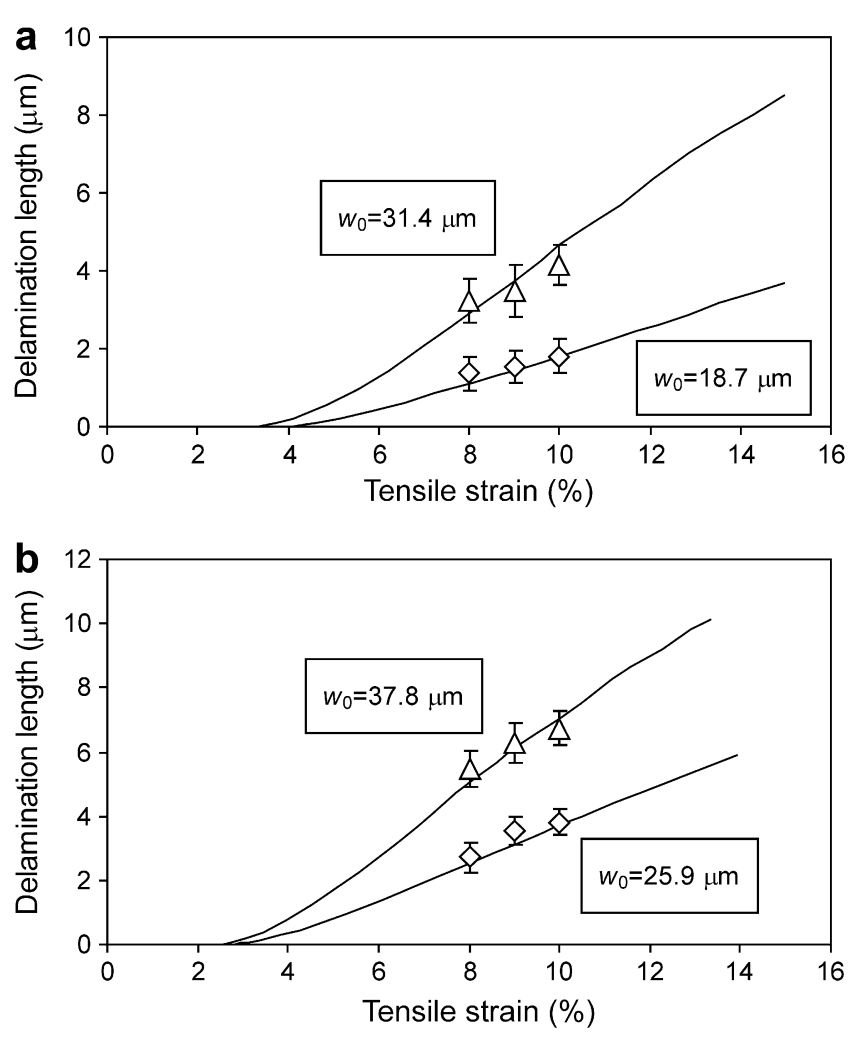

Fig. 8. Experimental (dots) and calculated (lines) edge delamination length of: (a) $600 \mathrm{~nm}$ and (b) $800 \mathrm{~nm}$ thick fragments with different widths $w_{0}$.

with the reduction in coating thickness, in qualitative agreement with the experimental observations. For example, the predicted delamination length at $10 \%$ strain for a fragment $400 \mathrm{~nm}$ thick and $23 \mu \mathrm{m}$ wide is $2.5 \mu \mathrm{m}$, which is close to the experimental data. For a fragment $200 \mathrm{~nm}$ thick and $11 \mu \mathrm{m}$ wide, the predicted delamination at the same strain equals $0.5 \mu \mathrm{m}$, which is below the resolution of the optical microscope.

According to the FEM analysis, the angle between the delaminated coating and the substrate does not exceed $10^{\circ}$. This result validates the layered media model in Appendix A used for interference pattern analysis. The separation between the coating and the substrate at the delamination front, evaluated by FEM, exceeds $20 \mathrm{~nm}$, which should provide a marked contrast in the intensity of reflected light as seen in Fig. 5. Therefore, it can be concluded that the geometry of the edge delamination predicted by FEM is consistent with the presence of an interference fringe. This result also validates the assumption that the abrupt change in brightness of the reflected light, observed along fragment edges during tests, closely approximates the location of the front of edge delamination.

Note that the selection of the cohesive zone parameters may be non-trivial in the considered case of an elastic-plastic substrate. The extent of substrate yielding associated with the propagation of delamination is affected by the strength of the cohesive elements (see e.g., Ref. [31]). The strength of the element is also indirectly related to the extent of the fracture process zone. For an interfacial ERR of $30 \mathrm{~J} \mathrm{~m}^{-2}$ and $\tau=200 \mathrm{MPa}$, the maximum sliding displacement of the element reaches $300 \mathrm{~nm}$ according to Eq. (7). In this case the size of the fracture process zone is $\sim 1 \mu \mathrm{m}$ or larger, which is comparable with the observed values of the length of delamination. A higher strength of cohesive elements at a fixed interfacial ERR would lead to smaller $\delta$ and hence a shorter fracture process zone. Fig. 9 shows the results of simulations for a fragment $800 \mathrm{~nm}$ thick and $37.8 \mu \mathrm{m}$ wide and for different interface strength values at a fixed ERR and an applied strain of $8 \%$. The lower curve plots the predicted delamination length - the distance from fragment edge to the crack front, i.e., the location where tractions on cohesive element faces are reduced to zero. The upper curve shows the position with maximum tractions on the crack faces (i.e., the beginning of the fracture process zone). It is seen that the length of the process zone (equal to the offset of the curves) exceeds $1 \mu \mathrm{m}$ for an interface strength below $300 \mathrm{MPa}$. Simulations with an interface strength of $400 \mathrm{MPa}$ or higher lead to the fracture process zone being localized in a smaller region, but the length of delamination in this case is significantly lower than that for a weaker interface. A larger fraction of the available ERR is spent on the plastic dissipation within the substrate in the case of a strong interface, therefore less energy remains available for crack propagation. The analysis of energy redistribution between decohesion and yielding for a crack propagating in an elastic-plastic material has been provided in Refs. [16,17].

It follows from the above discussion that, as could be expected in the case of a yielding polymer substrate, both the adhesive ERR and the strength are important interface parameters. It was demonstrated that a set of these two parameters, determined for mode II loading by means of numerical analysis of fragmentation test results, provides accurate description of the edge delamination process for differing fragment geometries.

\section{Conclusions}

$\mathrm{SiN}_{x} / \mathrm{PI}$ substrate with coating thicknesses ranging from $100 \mathrm{~nm}$ to $800 \mathrm{~nm}$ was fragmentation-tested under uniaxial

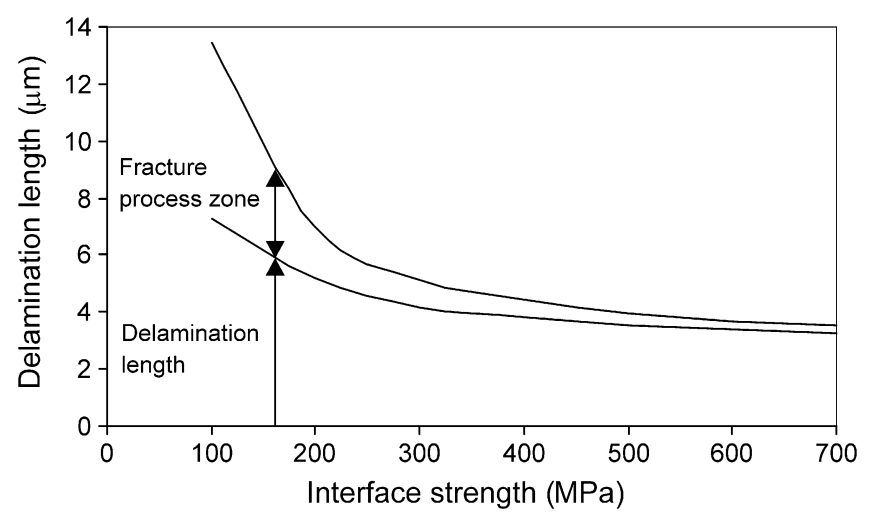

Fig. 9. Predicted delamination length as a function of the interface element strength at a fixed interfacial ERR. The lower and upper curves correspond to crack front and fracture process zone front, respectively. 
tension up to $15 \%$ nominal strain. Upon an initial stage of intensive segmentation cracking, decohesion developed in the coating fragments in the form of buckling-driven delaminations and edge delaminations. The latter initiated at lower strains for thicker coatings, and, for a coating of given thickness, for wider fragments. The evolution of edge delaminations with load was quantitatively characterized for the thicker coatings and wider fragments developing larger delaminations. The length of the edge delamination was correlated to the length of the bright interference fringe detected along the fragment edges observed under white light. The debonding process was modelled by FEM incorporating a cohesive zone at the front of the interfacial crack. The edge delaminations were found to be driven by mode II loading. Estimates of mode II critical interfacial ERR at $30 \mathrm{~J} \mathrm{~m}^{-2}$ and shear strength at $250 \mathrm{MPa}$ were obtained by fitting the predicted delamination evolution to the experimental data of coatings $600 \mathrm{~nm}$ and $800 \mathrm{~nm}$ thick. It was demonstrated that the combination of interferometry and numerical analysis based on a cohesive zone model provided an accurate description of the edge delamination process for differing fragment geometries.

\section{Acknowledgements}

Part of this work was supported by the EC-funded integrated Project FlexiDis (Contract IST-2004-4354). The authors acknowledge Unaxis France, Display Technology for the supply of film samples.

\section{Appendix A}

\section{A.1. The reflection of light from thin films}

The reflection of a light beam from a thin film can be analyzed using Fresnel coefficients [32]. When a light wave meets an interface between medium 1 and medium 2 with optical properties $n_{1}$ and $n_{2}$, respectively, part of the wave is reflected back into medium 1 , and part is transmitted into medium 2. The Fresnel reflection and transmission coefficients are (here and further only the normal incidence of the light beam is considered):

$r_{12}=\frac{n_{1}-n_{2}}{n_{1}+n_{2}} ; \quad t_{12}=\frac{2 n_{1}}{n_{1}+n_{2}}$

where $n_{1}$ and $n_{2}$ in general are complex numbers $n-\mathrm{i} k$ with refraction index $n$ and extinction coefficient $k$. For the thin film of medium 2 sandwiched between media 1 and 3, the ray transmitted through interface $1-2$ is partially reflected back at interface $2-3$. This ray is reflected back and forth as shown in Fig. 10a. The reflection coefficient can be obtained by summation of amplitudes of all the reflected rays, taking into account the geometric path difference and corresponding phase difference $-2 \theta$ between any two successive reflections. The phase difference $\theta$ is given by

$\theta=\frac{2 \pi}{\lambda} d n_{2}$
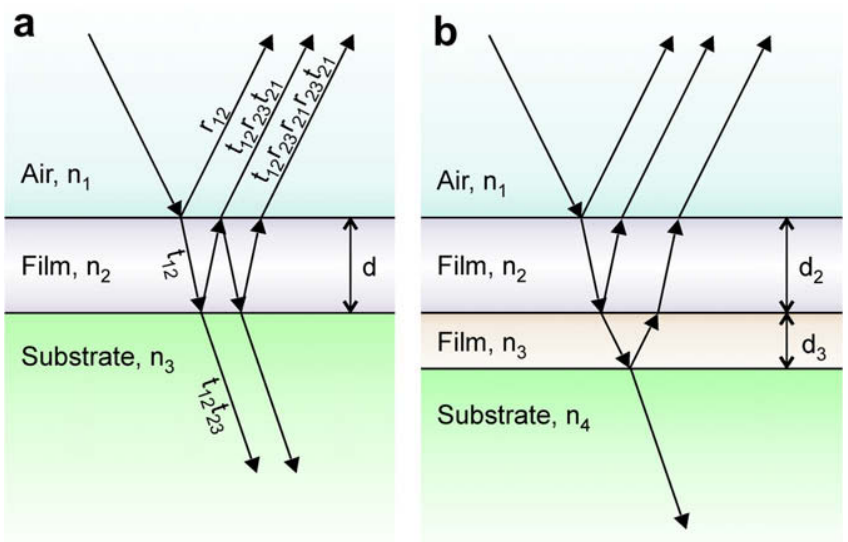

Fig. 10. Reflection of a light beam from thin film: (a) single layer and (b) two-layer coating.

where $\lambda$ is light wavelength and $d$ is thickness of the film. The reflection coefficient of a single layer can be easily obtained analytically (Airy formula) [32]:

$$
\begin{aligned}
r & =r_{12}+t_{12} r_{23} t_{21} e^{-2 i \theta}+t_{12} r_{23} r_{21} r_{23} t_{21} e^{-4 i \theta}+\cdots \\
& =r_{12}+\frac{t_{12} r_{23} t_{21} e^{-2 i \theta}}{1-r_{12} r_{23} e^{-2 i \theta}}=\frac{r_{12}+r_{23} e^{-2 i \theta}}{1+r_{12} r_{23} e^{-2 i \theta}}
\end{aligned}
$$

For the multilayered medium, the matrix method is often employed. The reflectance of the two-layer coating (Fig. 10b) can be analyzed by generalization of Eq. (A.3):

$$
r=\frac{r_{12}+r_{234} e^{-2 i \theta_{2}}}{1+r_{12} r_{234} e^{-2 i \theta_{2}}}
$$

where $r_{234}$ is the reflection coefficient of the composite structure consisting of a layer of medium 3 and an infinite medium 4 , and is given by

$r_{234}=\frac{r_{23}+r_{34} e^{-2 i \theta_{3}}}{1+r_{23} r_{34} e^{-2 i \theta_{3}}}$

and

$\theta_{2}=\frac{2 \pi}{\lambda} d_{2} n_{2} ; \quad \theta_{3}=\frac{2 \pi}{\lambda} d_{3} n_{3}$

where $n_{2}, n_{3}$ and $d_{2}, d_{3}$ are the refractive indices and thicknesses of layers 2 and 3, respectively, and $r_{12}, r_{23}$ and $r_{34}$ are the Fresnel coefficients of the corresponding interfaces.

The intensity of the reflected light can be found by integrating the reflectance over the visible light range, taking into account the spectrum $I_{0}$ of the incident light:

$I=\int I_{0}(\lambda) R(\lambda) d \lambda$

where the reflectance $R(\lambda)$ is given by $R=|r|^{2}$.

\section{References}

[1] Volinsky AA, Moody NR, Gerberich WW. Acta Mater 2002;50:441-66.

[2] Gerberich WW, Cordill MJ. Rep Prog Phys 2006;69:2157-203.

[3] Leterrier Y. Prog Mater Sci 2003;48:1-55. 
[4] Leterrier Y, Boogh L, Andersons J, Månson J-AE. J Polym Sci Part B: Polym Phys 1997;35:1449-61.

[5] Andersons J, Tarasovs S, Leterrier Y. Thin Solid Films 2009;517: 2007-11.

[6] Frank S, Handge UA, Olliges S, Spolenak R. Acta Mater 2009;57:1442-53.

[7] Chiu SL, Leu J, Ho PS. J Appl Phys 1994;76:5136-42.

[8] Leu J, Ho PS, Chiu SL. J Appl Phys 1994;76:5143-8.

[9] Alaca BE, Saif MTA, Sehitoglu H. Acta Mater 2002;50: 1197-209.

[10] Andersons J, Leterrier Y, Tornare G, Dumont P, Månson J-AE. Mech Mater 2007;39:834 44.

[11] Leterrier Y, Månson J-AE. J Mater Sci Lett 1997;16:120-1.

[12] Jansson NE, Leterrier Y, Medico L, Månson J-AE. Thin Solid Films 2006;515:2097-105.

[13] Yu HH, He MY, Hutchinson JW. Acta Mater 2001;49:93-107.

[14] Yu H, Hutchinson JW. Thin Solid Films 2003;423:54-63.

[15] Wei Y, Hutchinson JW. J Mech Phys Solids 1997;45:1137-59.

[16] Shet C, Chandra N. J Eng Mater Technol 2002;124:440-50.

[17] Chandra N, Shet C. CMES 2004;5:21-33.

[18] George M, Coupeau C, Colin J, Grilhé J. Thin Solid Films 2003;429:267-72.

[19] McGuigan AP, Huey BD, Briggs GAD, Kolosov OV, Tsukahara Y, Yanaka M. Appl Phys Lett 2002;80:1180-2.

[20] De Boer MP, Michalske TA. J Appl Phys 1999;86:817-27.

[21] Bhate D, Dunn ML. Microelectr Reliab 2007;47:2014-24.
[22] El amrani A, Menous I, Mahiou L, Tadjine R, Touati A, Lefgoum A. Renew Energy 2008;33:2289-93.

[23] Lee Jaehyeong, Lakshminarayan N, Dhungel Suresh Kumar, Kim Kyunghae, Yi Junsin. Solar Energy Mater Solar Cells 2009;93: 256-61.

[24] Coates K, Morrison S, Narayanan S, Madan A. Deposition of silicon nitride to improve the conversion efficiency of multicrystalline silicon solar cells. In: Proc 16th European photovoltaic solar energy conf, Glasgow; 2000. p. 1279.

[25] Dijken AV, Toonder JD, Bouten P. Nano indentations on inorganic coatings for the FlexiDis project. Technical report, Philips Research Laboratories, Eindhoven, The Netherlands; 2005.

[26] Jiang M, Wood NO, Komanduri R. Wear 1998;220:59-71.

[27] Andersons J, Modniks J, Leterrier Y, Tornare G, Dumont P, Månson J-AE. Theor Appl Fract Mech 2008;49:151-7.

[28] Song GM, Sloof WG, Pei YT, De Hosson JThM. Surf Coat Technol 2006;201:4311-6.

[29] Pasquier AD, Miller S, Chhowalla M. Solar Energy Mater Solar Cells 2006;90:1828-39.

[30] Alfano G. Compos Sci Technol 2006;66:723-30.

[31] van der Sluis O, Yuan CA, van Driel WD, Zhang GQ. Advances in delamination modeling. In: Morris JE, editor. Nanopackaging. Nanotechnologies and electronics packaging. Heidelberg: Springer; 2008. p. 61-92.

[32] Yeh P. Optical waves in layered media. Hoboken (NJ): WileyInterscience; 2005. 